\title{
An adjoint-based approach for a class of nonlinear Fokker-Planck equations and related systems
}

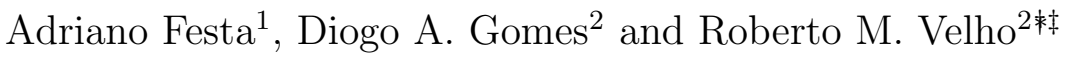

April 23, 2018

\begin{abstract}
Here, we introduce a numerical approach for a class of Fokker-Planck (FP) equations. These equations are the adjoint of the linearization of Hamilton-Jacobi (HJ) equations. Using this structure, we show how to transfer properties of schemes for HJ equations to FP equations. Hence, we get numerical schemes with desirable features such as positivity and mass-preservation. We illustrate this approach in examples that include mean-field games and a crowd motion model.
\end{abstract}

\section{Introduction}

Fokker-Plank (FP) equations model the time evolution of a probability density. The general set up is as follows. Given an open subset of $\mathbb{R}^{d}, \Omega$, a terminal time, $T>0$, and a (drift) vector field, $b(x, t): \Omega \times[0, T] \rightarrow \Omega$, we seek to find a time-dependent probability distribution, $\rho: \Omega \times[0, T] \rightarrow \mathbb{R}$, solving

$$
\begin{cases}\partial_{t} \rho-\varepsilon \Delta \rho+\operatorname{div}(b(x, t) \rho)=0 & \text { in } \Omega \times[0, T], \\ \rho(\cdot, 0)=\rho_{0}(\cdot) & \text { in } \Omega .\end{cases}
$$

Also, we supplement the above problem with boundary conditions on $\partial \Omega \times[0, T]$, where $\partial \Omega$ is the boundary of $\Omega$. We are particularly interested in problems where $b$ may depend on $\rho$ either directly or through an unknown function determined by an additional partial differential equation. Two examples discussed in this paper are the forward-forward mean-field game (MFG) and the Hughes model.

The Fokker-Planck equation was introduced in statistical mechanics. This equation has multiple applications in economics [24, 28], crowd motion models

${ }^{* *}$ D. Gomes was partially supported by KAUST baseline and start-up funds and KAUST SRI, Uncertainty Quantification Center in Computational Science and Engineering. A. Festa was partially supported by the Haute-Normandie Regional Council via the M2NUM project

${ }^{\dagger}$ Institut National de Sciences Appliquées, LMI lab Avenue de l'Université, 76800 SaintÉtienne-du-Rouvray, Rouen, France adriano.festa@insa-rouen.fr

$\ddagger 2$ King Abdullah University of Science and Technology (KAUST), CEMSE Division, Thuwal 23955-6900. Saudi Arabia, and KAUST SRI, Center for Uncertainty Quantification in Computational Science and Engineering. diogo.gomes@kaust.edu.sa, roberto.velho@gmail.com 
[25, 27, 34], and biological models [14, 23]. Due to the complex structure of those equations, the computation of explicit solutions is not possible. Hence, effective numerical methods for approximating solutions of FP equations have a broad interest.

Here, we propose a technique to obtain approximation schemes for FP equations using their representation as the adjoint of the linearization of HamiltonJacobi (HJ) equations. In this way, all monotone numerical schemes proposed in the context of HJ equations give rise to consistent schemes for FP equations. In particular, as required by the nature of the problem, these schemes preserve positivity and are conservative, i.e., under suitable boundary conditions mass is preserved.

Previously, the adjoint structure of the FP equation was used by several authors, for example, in [1] and in [2]. In those references, the authors propose a finite-difference scheme which is the adjoint of the linearization of the upwind scheme used to approximate a convex Hamiltonian. In $[9,10,11,12,13]$, the authors propose a semi-Lagrangian numerical method using a slightly different procedure, but based on a similar principle.

The main contribution of the present paper is to show how to use the adjoint structure with a wide class of numerical solvers, and without limitations on the problem dimension. Here, in contrast to the above references, we develop semi-discrete schemes, where the spatial variable is discretized. To construct the semi-discretization, we apply symbolic calculus to assemble the schemes by exact formula manipulation. The evolution in time corresponds to a system of ordinary differential equations (ODE). These can be solved with different methods, depending on the smoothness of the solution and desired accuracy. The semi-discrete schemes obtained symbolically are compiled before the numerical simulation of the resulting system of ODEs, reducing substantially both the computational time and the work of writing the code of such schemes.

Outline of the paper. We end this introduction with an outline of this paper. The adjoint structure is examined in Section 2. Next, in Section 3, we proof key features of the method: positivity and mass-conservation. In Section 4, we describe our numerical framework, its properties, and discuss sample schemes. Finally, in Section 5, consider some problems where our framework applies. These included mean-field games and a crowd motion model.

\section{Adjoint structure}

The relation between a FP equation and its adjoint equation is well known. In recent works, $[5,6,7,8,15,20,35]$, this relation was used to study regularity properties, vanishing viscosity limits, and rates of convergence of numerical methods. Those results are based on the observation that a FP equation is the adjoint of the linearization of a certain HJ equation.

\subsection{Linearization and duality}

Here, we discuss the relation between FP and HJ equations. First, we consider the HJ operator

$$
H J(u):=-u_{t}(x, t)+H(x, D u(x, t))-\varepsilon \Delta u(x, t),
$$


with the Hamiltonian $H=H(x, p): \Omega \times \mathbb{R}^{d} \rightarrow \mathbb{R}, \Omega \subset \mathbb{R}^{d}$. Further, we define the nonlinear generator

$$
A^{H J} u:=H(x, D u(x, t))-\varepsilon \Delta u(x, t) .
$$

Here, we write $D u=D_{x} u$ for the gradient in the variable $x=\left(x_{1}, \cdots, x_{d}\right)$. The parameter $\varepsilon$ is called the viscosity.

To linearize (2.1) around a generic point $u_{0}$, we expand $u=u_{0}+\lambda w$, then take the derivative in $\lambda$, and, finally, consider the limit $\lambda \rightarrow 0$. Now, we compute this linearization. Boundary conditions are discussed in the next subsection.

The expansion $H J\left(u_{0}+\lambda w\right)$ gives

$$
\begin{aligned}
-\partial_{t}\left(u_{0}+\lambda w\right)+H & \left(x, D\left(u_{0}+\lambda w\right)\right)-\varepsilon \Delta\left(u_{0}+\lambda w\right) \\
& =-\left(u_{0}\right)_{t}-\lambda w_{t}+H\left(x, D u_{0}+\lambda D w\right)-\varepsilon \Delta u_{0}-\lambda \varepsilon \Delta w .
\end{aligned}
$$

We suppose $H$ has enough regularity so that we can take the derivative of the preceding expression with respect to $\lambda$. Next, we let $\lambda \rightarrow 0$, obtaining the operator

$$
L(w):=-w_{t}+D_{p} H\left(x, D u_{0}\right) \cdot D w-\varepsilon \Delta w,
$$

the linearization of the HJ operator. The (linear) generator of $L$ is

$$
A^{L} w:=D_{p} H\left(x, D u_{0}\right) \cdot D w-\varepsilon \Delta w .
$$

Finally, we compute the adjoint of $L$ by integration by parts. We fix smooth functions, $w$ and $\rho$, and derive the identity

$$
\begin{aligned}
& \iint_{[0, T] \times \Omega}\left(-w_{t}+D_{p} H\left(x, D u_{0}\right) \cdot D w-\varepsilon \Delta w\right) \rho \\
& =\iint_{[0, T] \times \Omega}\left(\rho_{t}-\operatorname{div}_{x}\left(D_{p} H\left(x, D u_{0}\right) \rho\right)-\varepsilon \Delta \rho\right) w \\
& +\iint_{[0, T] \times \partial \Omega}\left(D_{p} H\left(x, D u_{0}\right)\right) \cdot n \rho w+\varepsilon \frac{\partial \rho}{\partial n} w-\varepsilon \rho \frac{\partial w}{\partial n} \\
& -\int_{\Omega} \rho(x, T) w(x, T)-\rho(x, 0) w(x, 0),
\end{aligned}
$$

where $n$ is the normal vector to the boundary, $\partial \Omega$. The last calculation shows that the adjoint of $L$ is the following $\mathrm{FP}$ operator

$$
L^{*} \rho:=\rho_{t}-\operatorname{div}_{x}\left(D_{p} H\left(x, D u_{0}\right) \rho\right)-\varepsilon \Delta \rho,
$$

whose generator is $A^{F P} \rho:=-\operatorname{div}_{x}\left(D_{p} H\left(x, D u_{0}\right) \rho\right)-\varepsilon \Delta \rho$.

\subsection{Boundary conditions}

Now, we address the boundary conditions for $(1.1)$ on $\partial \Omega \times[0, T]$. The discussion of initial conditions is straightforward. Two common boundary conditions for FP equations are Dirichlet data and a prescribed flow via Neumann conditions. 
In the Dirichlet case, the data vanishes on the boundary. This corresponds to the case where particles exit once they reach the boundary. The prescribed flow case represents a current of particles or agents crossing the boundary. Thus, with a zero flow, the mass is conserved.

Both the Dirichlet condition and the zero flow Neumann condition determine cancellations in the boundary integrals in (2.3). This suggests different conditions for the HJ operator, its linearized version, and its adjoint, the FP operator.

The first case corresponds to a FP equation with Dirichlet boundary conditions:

$$
\left\{\begin{array}{lr}
\rho_{t}(x, t)-\operatorname{div}\left(D_{p} H(x, D u) \rho\right)=\varepsilon \Delta \rho, & \text { in } \Omega \times[0, T], \\
\rho(\cdot, t)=0, & \text { on } \partial \Omega \times[0, T] .
\end{array}\right.
$$

We consider the HJ operator with the boundary conditions

$$
\left\{\begin{array}{l}
-u_{t}(x, t)+H(x, D u(x, t))-\varepsilon \Delta u(x, t), \text { in } \Omega \times[0, T], \\
u(\cdot, t)=g_{1}(\cdot, t), \text { for any } g_{1}, \quad \text { on } \partial \Omega \times[0, T],
\end{array}\right.
$$

and the linearized operator as

$$
\left\{\begin{array}{lr}
-w_{t}+D_{p} H(x, D u) \cdot D w-\varepsilon \Delta w, & \text { in } \Omega \times[0, T], \\
w(\cdot, t)=0, & \text { on } \partial \Omega \times[0, T] .
\end{array}\right.
$$

The second case corresponds to a FP equation with a flux through the boundary

$$
\begin{cases}\rho_{t}(x, t)-\operatorname{div}\left(D_{p} H(x, D u) \rho\right)-\varepsilon \Delta \rho(x, t)=0 & \text { in } \Omega \times[0, T], \\ D_{p} H(x, D u) \cdot n \rho+\varepsilon \frac{\partial \rho}{\partial n}(x, t)=g_{2}(x, t), & \text { on } \partial \Omega \times[0, T],\end{cases}
$$

where $g_{2}$ is the desired in/out-flow through $\partial \Omega$. We can consider diverse boundary conditions for the HJ operator: Dirichlet type, state-constraint, reflection at the boundary, and Neumann type. In the following example, we use Neumann conditions with zero flow. The Hamilton-Jacobi operator is

$$
\left\{\begin{array}{lr}
-u_{t}(x, t)+H(x, D u(x, t))-\varepsilon \Delta u(x, t), & \text { in } \Omega \times[0, T], \\
\frac{\partial u}{\partial n}(x, t)=0, & \text { on } \partial \Omega \times[0, T],
\end{array}\right.
$$

with the corresponding linearization

$$
\left\{\begin{array}{lr}
-w_{t}+D_{p} H(x, D u) \cdot D w-\varepsilon \Delta w, & \text { in } \Omega \times[0, T], \\
\frac{\partial w}{\partial n}(\cdot, t)=0, & \text { on } \partial \Omega \times[0, T] .
\end{array}\right.
$$

In the schemes we describe in section 4 , we focus on the spatial discretization of the previous operators. The time discretization can be chosen separately, depending on the application. This is the reason we simplified the discussion above and we avoided initial conditions, which are straightforward.

We now discuss the connection between stochastic differential equations with their density formulation, the Fokker-Planck equation, and then the associated Hamiltonian, via the adjoint structure. This pathwise interpretation given by 
the solution of the SDE provides understanding on how to treat boundary conditions.

A nonlinear FP equation is related to the solution of a stochastic differential equation of McKean-Vlasov type (or mean-field type), see [29, 30, 31, 33]. More precisely, we consider the stochastic differential equation (SDE)

$$
\left\{\begin{array}{l}
d X(t)=b(X(t), \rho(X(t), t), t) d t+\sqrt{2 \varepsilon} d W(t), \\
X(0)=X^{0},
\end{array}\right.
$$

where $b: \mathbb{R}^{d} \times \mathbb{R}_{+} \times \mathbb{R}_{+} \rightarrow \mathbb{R}^{d}$ is a regular vector-valued function, $X^{0}$ is a random vector in $\mathbb{R}^{d}$, independent of the Brownian motion $W(\cdot)$, with density $\rho_{0}$, and $\rho(\cdot, t)$ is the density of $X(t)$. It can be shown (see [26]) that under suitable growth and regularity conditions for $b(2.5)$ admits a unique solution and $\rho$ is the unique classical solution of the nonlinear FP equation

$$
\partial_{t} \rho-\varepsilon \Delta \rho+\operatorname{div}(b(x, \rho, t) \rho)=0 .
$$

With Dirichlet conditions, those trajectories end at the boundary; for zeroflux conditions, they are reflected, see [3, 19]. Moreover, the Hamilton-Jacobi operator from which $(2.2)$ can be derived is

$$
-u_{t}-\varepsilon \Delta u-b(x, \rho, t) \cdot D u .
$$

Remark 2.1. Our methods can be extended to study stationary FP equations. In this case, the associated Hamilton-Jacobi operator is stationary.

\section{Properties}

In this section, we use a duality argument to obtain properties of FP equations from corresponding properties of the evolution semigroup associated with a HJ equation. The arguments detailed here are valid without any substantial changes for the discretized problems using the semigroups associated with the discretized equation. This discretization can be performed in multiple ways, including finite-differences and semi-Lagrangian schemes.

We denote by $\langle f, g\rangle=\int_{\Omega} f g$ the duality product, and by $S_{t}$ the semigroup corresponding to the evolution in time given by the linearized operator (2.2). This semigroup preserves order; that is $v \leqslant w$ implies $S_{t} v \leqslant S_{t} w$. Moreover, we assume that $S_{t} 1=1$. We note that the property $S_{t} 1=1$ depends on the boundary conditions for (2.2). We define the adjoint $S_{t}^{*}$ of $S_{t}$ by

$$
\left\langle S_{t}^{*} u, v\right\rangle=\left\langle u, S_{t} v\right\rangle .
$$

We have then the following results:

Proposition 3.1 (Positivity). Suppose $S_{t}$ is monotone. Then, the evolution of the initial density $\rho_{0}$ through the adjoint semigroup, $S_{t}^{*}$, preserves positivity. That is, if $\rho_{0} \geqslant 0$, we have $S_{t}^{*} \rho \geqslant 0$, for all $t \in[0, T]$.

Proof: Denote by $w_{T}$ the terminal condition for the linearized operator. First, note that $w_{T} \geqslant 0$ implies $S_{t} w_{T} \geqslant 0$. This follows from the maximum principle for HJ equations. Thus, for $w_{T} \geqslant 0$, we have

$$
\left\langle S_{t}^{*} \rho, w_{T}\right\rangle=\left\langle\rho, S_{t} w_{T}\right\rangle \geqslant 0,
$$


since $\rho \geqslant 0$, and $S_{t} w_{T} \geqslant 0$. Accordingly, $S_{t}^{*} \rho \geqslant 0$.

Now, we show that if $S_{t} 1=1$ the mass is conserved by $S_{t}^{*}$. For example, under periodic boundary conditions, the evolution of (2.2) preserves constants. This is not the case under Dirichlet boundary conditions, where mass loss through the boundary occurs.

Proposition 3.2 (Conservation of Mass). Suppose $S_{t} 1=1$. Let $\rho_{0}$ be the initial density probability distribution, i.e. $\int_{\Omega} \rho_{0}=1$. Then, for all $t \in[0, T]$, the evolution of this probability measure through the adjoint semigroup, $S_{t}^{*} \rho_{0}$, is also a probability measure.

Proof: First, observe that $S_{t} 1=1$. Then,

$$
\int_{\Omega} S_{t}^{*} \rho_{0}=\left\langle S_{t}^{*} \rho_{0}, 1\right\rangle=\left\langle\rho_{0}, S_{t} 1\right\rangle=\left\langle\rho_{0}, 1\right\rangle=\int_{\Omega} \rho_{0}=1 .
$$

Remark 3.3. The preceding assumptions on $S_{t}$ are not restrictive for our applications. For discrete problems, the linearized semigroups $S_{t}$ for HJ equations are usually monotone and, in cases where mass conservation for the FP equation holds, also satisfy $S_{t} 1=1$. Often, it is easier to check monotonicity and that $S_{t} 1=1$ than the corresponding properties for $S_{t}^{*}$.

Remark 3.4. If the viscosity vanishes ( $\varepsilon=0$ ), our approach is still valid for classical solutions (for first-order equations and systems regularity may fail and our computations need to be justified case by case). A first-order HJ operator gives rise to a continuity equation $(\mathrm{CE})$, i.e. a $\mathrm{FP}$ equation without viscosity. This case is considered in section 5 , where we extend our numerical scheme to address systems of partial differential equations (PDEs). Those systems arise in multiple applications such as MFGs, population models, traffic flow problems, and modeling in chemotaxis.

\section{Numerical approach}

Our numerical approach relies on the relation between the HJ framework and the corresponding adjoint FP equation. Once we choose a semi-discrete (discrete in space) numerical scheme for (2.1), we can reuse it to construct an approximation for (2.4).

Before proceeding, we define additional notation. To simplify, we consider a scheme for the case where the domain $\Omega$ is $\mathbb{T}^{2}$ (2-D torus). Let $\mathbb{T}_{\Delta x}^{2}$ be an uniform grid on $\mathbb{T}^{2}$, with constant discretization parameter $\Delta x>0$. Let $x_{i, j}$ denote a generic point in $\mathbb{T}_{\Delta x}^{2}$. The space of grid functions defined on $\mathbb{T}_{\Delta x}^{2}$ is denoted by $\mathcal{G}\left(\mathbb{T}_{\Delta x}^{2}\right)$, and the functions $U, M \in \mathcal{G}\left(\mathbb{T}_{\Delta x}^{2}\right)$ (approximations of respectively $u$ and $\rho$ ) are called $U_{i, j}$ and $M_{i, j}$, when evaluated at $x_{i, j}$. We utilize a semi-discrete numerical scheme $N(x, p): \mathbb{T}_{\Delta x}^{2} \times \mathbb{R}^{d} \rightarrow \mathbb{R}$ monotone and consistent to approximate the operator $H(x, p)$ by the discrete operator

$$
N(x, \mathcal{D} U)
$$


where $\mathcal{D} U$ is a discretization of the gradient operator on $U$. Thanks to the adjoint structure, we use the scheme (4.1) to assemble a discrete operator $K$

$$
K(x, \mathcal{D} U, M):=\left(D_{U} N(x, \mathcal{D} U)\right)^{T} M,
$$

that discretizes the spatial part of the FP operator (2.4). The discrete approximation $M$ for the solution of the FP equation is then given by the ODE

$$
M_{t}-K(x, \mathcal{D} U, M)-\varepsilon \Delta_{d} M=0,
$$

or simply,

$$
M_{t}-\left(D_{U} N(x, \mathcal{D} U)\right)^{T} M-\varepsilon \Delta_{d} M=0,
$$

i.e., a discrete equivalent to the adjoint structure seen in (2.4). Here, the nonlinear part of the operator corresponds to the discrete operator $D_{U} N\left(x_{i, j}, \mathcal{D} U\right)$, and $\Delta_{d} M$ is a discretization of the Laplacian, which is added to the scheme to increase the stability, if necessary.

We note that the operators $N$ and $K$ depend on the monotone approximation scheme used to discretize the HJ equation. The operator $K$ can be computed using a symbolic differentiation as we show in section 5.1. Also, the Hamiltonian $H(x, p)$ must be sufficiently regular in the $p$ variable so that the scheme is properly defined. The properties of positivity and mass conservation are valid at the discrete level as consequence of the semigroup arguments in section 3 .

We now prove how the consistency of schemes for HJ equations transfers to schemes for FP equations.

Proposition 4.1 (Consistency). Suppose $u$ is the solution to a linerized HJ equation and $\rho$ the solution to the associated FP equation. Also, suppose that $u$ and $\rho$ are $C^{\infty}$, and consider their restriction to the grid points. Denote by $S_{t}^{N}$ the linearized semigroup corresponding to a discretization of the HJ equation with $o(1)$ error, i.e.,

$$
S_{t}^{N} u=S_{t} u+o(1)
$$

with $S_{t}$ as in the previous sections. Then, the adjoint semigroup $\left(S_{t}^{N}\right)^{*}$ operating on the discretization of the FP equation possesses the same order of error, i.e.,

$$
\left(S_{t}^{N}\right)^{*} \rho=\left(S_{t}\right)^{*} \rho+o(1) .
$$

Proof: By the adjoint structure between the HJ and FP equations, and the hypothesis of consistency in equation (4.4), we have

$$
\left\langle\left(S_{t}^{N}\right)^{*} \rho, u\right\rangle=\left\langle\rho, S_{t}^{N} u\right\rangle=\left\langle\rho, S_{t} u\right\rangle+\langle\rho, o(1)\rangle=\left\langle S_{t}^{*} \rho, u\right\rangle+o(1),
$$

which proves (4.5).

Remark 4.2 (Convergence). We stress that the main novelty in the current paper is a systematic approach to build schemes for FP equations from schemes for HJ equations. Naturally, different schemes will have different convergence properties, which must be examined case by case. Furthermore, to address the convergence of a method, we must know the regularity of the vector field in the FP equation and the existence of a solution. In the numerical simulations developed in section 5 , this regularity is not always known. Also, for the crowd motion problem we consider, the existence of solutions is not known. 


\subsection{Finite differences}

Now, we consider an explicit scheme using our method. We describe an upwind discretization for the Hamiltonian, which we assume to be

$$
H(x, p)=g(x)+|p|^{\alpha}, \text { with } \alpha>1 \text {, and } p=\left(p_{1}, p_{2}, p_{3}, p_{4}\right) .
$$

We define the standard finite-difference operators as

$$
\left(\mathcal{D}_{1}^{ \pm} u\right)_{i, j}=\frac{u_{i \pm 1, j}-u_{i, j}}{\Delta x}, \quad\left(\mathcal{D}_{2}^{ \pm} u\right)_{i, j}=\frac{u_{i, j \pm 1}-u_{i, j}}{\Delta x},
$$

and

$$
\Delta_{d} u=\frac{1}{\Delta x^{2}}\left(4 u_{i, j}-u_{i+1, j}-u_{i, j+1}-u_{i-1, j}-u_{i, j-1}\right) .
$$

The approximation of the operator $H(x, p)$ is

$$
N(x, p)=g(x)+G\left(p_{1}^{-}, p_{2}^{+}, p_{3}^{-}, p_{4}^{+}\right),
$$

where for a real number $r$, we define the operators

$$
r^{+}:=\max (0, r), \quad r^{-}:=\max (0,-r),
$$

and

$$
G(p)=G\left(p_{1}, p_{2}, p_{3}, p_{4}\right):=\left(p_{1}^{2}+p_{2}^{2}+p_{3}^{2}+p_{4}^{2}\right)^{\frac{\alpha}{2}} .
$$

The operators $r^{+}$and $r^{-}$are chosen to preserve the monotonicity of the scheme for the HJ operator, which is well defined backward in time. Plugging the finite differences operators in $N(x, p)$ we have

$$
\begin{aligned}
& N\left(x_{i, j},[\mathcal{D} U]_{i, j}\right)=g(x)+G\left(\left(\mathcal{D}_{1}^{+} u\right)_{i, j}^{-},\left(\mathcal{D}_{1}^{-} u\right)_{i, j}^{+},\left(\mathcal{D}_{2}^{+} u\right)_{i, j}^{-},\left(\mathcal{D}_{2}^{-} u\right)_{i, j}^{+}\right)= \\
& g(x)+\left[\left(\left(\mathcal{D}_{1}^{+} u\right)_{i, j}^{-}\right)^{2}+\left(\left(\mathcal{D}_{1}^{-} u\right)_{i, j}^{+}\right)^{2}+\left(\left(\mathcal{D}_{2}^{+} u\right)_{i, j}^{-}\right)^{2}+\left(\left(\mathcal{D}_{2}^{-} u\right)_{i, j}^{+}\right)^{2}\right]^{\frac{\alpha}{2}} .
\end{aligned}
$$

Now, we compute the operator $K(x, \mathcal{D} U, M)$, and we obtain

$$
\begin{aligned}
& K\left(x_{i, j},[\mathcal{D} U]_{i, j}, M_{i, j}\right)= \\
& \frac{1}{\Delta x}\left[M_{i, j} \frac{\partial N}{\partial p_{1}}\left(x_{i, j},[\mathcal{D} U]_{i, j}\right)-M_{i-1, j} \frac{\partial N}{\partial p_{1}}\left(x_{i-1, j},[\mathcal{D} U]_{i-1, j}\right)\right. \\
&+M_{i+1, j} \frac{\partial N}{\partial p_{2}}\left(x_{i+1, j},[\mathcal{D} U]_{i+1, j}\right)-M_{i, j} \frac{\partial N}{\partial p_{2}}\left(x_{i, j},[\mathcal{D} U]_{i, j}\right) \\
&+M_{i, j} \frac{\partial N}{\partial p_{3}}\left(x_{i, j},[\mathcal{D} U]_{i, j}\right)-M_{i, j-1} \frac{\partial N}{\partial p_{3}}\left(x_{i, j-1},[\mathcal{D} U]_{i, j-1}\right) \\
&\left.\quad+M_{i, j+1} \frac{\partial N}{\partial p_{4}}\left(x_{i, j+1},[\mathcal{D} U]_{i, j+1}\right)-M_{i, j} \frac{\partial N}{\partial p_{4}}\left(x_{i, j},[\mathcal{D} U]_{i, j}\right)\right]
\end{aligned}
$$

We then use this expression for the operator in (4.2).

This scheme coincides with the adaptation of the techniques proposed in [1] to our case. The advantage here is the fact that the properties of positivity 
and mass conservation are automatically obtained from the hypotheses on the discretization of the HJ operator; unlike typical schemes for FP equations, where such properties must be proved a posteriori. Analogously, in case we have chosen the semi-Lagrangian scheme for HJ equations previously presented, we would be creating a "dual semi-Lagrangian" method for FP equations. This is the main point in this work: the possibility of generating "dual methods" with desired properties for FP equations based on originally well-established methods for HJ equations. We explicit this possible method in the following section.

\subsection{Semi-Lagrangian scheme}

To describe a semi-Lagrangian scheme appropriate to approximate (4.6), we introduce the operator

$$
\mathcal{D}^{\gamma} U_{i, j}:=\max _{\gamma \in B(0,1)} \frac{\mathcal{I}[U]\left(x_{i, j}, \gamma\right)-U\left(x_{i, j}\right)}{h}
$$

where $B(0,1)$ is the unitary ball in $\mathbb{R}^{2}, h$ a parameter of the same order of $\sqrt{\Delta x}$, and

$$
\mathcal{I}[U]\left(x_{i, j}, \gamma\right)=\frac{1}{2} \sum_{i=1}^{2}\left(\mathbb{I}[U]\left(x_{i, j}+\gamma h+e_{i} \sqrt{2 \varepsilon h}\right)+\mathbb{I}[U]\left(x_{i, j}+\gamma h-e_{i} \sqrt{2 \varepsilon h}\right)\right)
$$

Here, $\mathbb{I}[u](x)$ is an interpolation operator on the matrix $U$, and $e_{i}$ is the $i$ unitary vector of an orthonormal basis of the space. Details on how to choose the interpolation operator are discussed in [16]. In our case, the discrete operator has the form

$$
N\left(x, \mathcal{D}^{\gamma} U_{i, j}\right):=g(x)+\left(\mathcal{D}^{\gamma} U_{i, j}\right)^{\alpha} .
$$

We take the adjoint of the linearized of $N$, by using (4.9), and we apply it to (4.2); analogously as performed for the finite-difference scheme. This scheme formally differs from the one proposed in [13], since the parameter $\gamma$ in (4.10) is computed in the dual formulation of the problem. We note that the operator $N(x, p)$ in $(4.11)$ is monotone by construction, see [16].

\section{Applications to systems of PDEs}

One immediate application of our numerical scheme is to solve "measure-potential" systems of PDEs. These systems comprise an equation for the evolution of a measure coupled with a second equation for a potential, or value function. Typically, this potential determines the drift for the convection in the first equation. Many problems have this structure: mean-field games, traffic-flow models, crowd motion, and chemotaxis.

Here, we describe how to use our method in the following examples: two 1-D forward-forward mean-field games (FFMFG) problems and a crowd motion model. All the simulations were performed on a $2.3 \mathrm{GHz}$ i7 computer with $16 \mathrm{~GB}$ of RAM. 


\subsection{Example: Hughes model in 1-D}

Now, we illustrate the application of our numerical approach to a model for crowd motion model due to Hughes [25]. We discuss this simple 1-D model with the intention of showing the steps connected to the adjoint structure, the discretization of the corresponding operators, and the extension of the methodology to measure-potential systems of PDEs.

The Hughes model comprises a FP equation, describing the evolution of the density of pedestrians/agents, coupled to an Eikonal (EK) equation that gives the optimal movement direction. This system is

$$
\left\{\begin{array}{l}
\rho_{t}(x, t)-\operatorname{div}\left(\rho(1-\rho)^{2} D u\right)=0 \\
|D u(x)|^{2}=\frac{1}{(1-\rho)^{2}},
\end{array}\right.
$$

together with an initial condition for the density and Dirichlet/Neumann boundary conditions. The goal is to exit a domain $\Omega$ in minimal time, taking into account congestion effects. Due to the stationary character of the EK equation, this system is not of MFG type. The density, $\rho$, evolves as if at each instant of time, the EK equation sees a frozen density. Then the agents choose the direction that leads to the shortest-time to evacuation, and this process determines the evolution of $\rho$.

We now describe how the Hughes system can be studied via our framework. Performing the same steps as in section 2, with the HJ operator

$$
-u_{t}+f(\rho) H(x, D u)-\varepsilon \Delta u
$$

where $f(\rho)$ is a regular function of the density, we obtain the associated FP equation

$$
\rho_{t}-\operatorname{div}\left(f(\rho) D_{p} H(x, D u) \rho\right)=\varepsilon \Delta u .
$$

By setting $f(\rho)=(1-\rho)^{2}$, and $H(x, p)=\frac{|p|^{2}}{2},(5.3)$ becomes the first equation of (5.1); and (5.2) is the adjoint operator we must study. More explicitly, starting by the spatial part of the HJ equation, the adjoint structure is as follows:

$$
(1-\rho)^{2} \frac{|D u|^{2}}{2} \stackrel{\text { Linearization }}{\longrightarrow}(1-\rho)^{2} D u \cdot D w \stackrel{\text { Adjoint }}{\longrightarrow}-\operatorname{div}\left[\rho(1-\rho)^{2} D u\right],
$$

where $u, w$, and $\rho$ follow the notation of section 2 .

We illustrate the use of finite differences to discretize the generator of the HJ operator. We choose the monotone scheme:

$$
N_{n}(u) \equiv\left(1-\rho_{n}\right)^{2}\left[\frac{\max \left\{u_{n}-u_{n-1}, 0\right\}^{2}}{2 h^{2}}+\frac{\max \left\{u_{n}-u_{n+1}, 0\right\}^{2}}{2 h^{2}}\right] .
$$

Once we discretize the domain $\Omega$ (a finite interval of $\mathbb{R}$ in this example), we calculate the previous discrete operators in each grid point, taking also in account the discretized versions of the boundary conditions. We have then a matrix, with the dimensions of the grid, whose entries have the expressions of the form of (5.4). The matrix entries (a vector in the 1-D case) have the $u_{j}$ 's 
( $j \in\{0,1, \ldots, P\}, P$ being the size of the grid in this $1-\mathrm{D}$ example) as a parameter. The numerical value of this matrix will only be evaluated once we have an approximation for the values of $u$. This will be done by solving the EK equation with an initial guess. Since the EK equation is a particular case of a HJ equation, we choose to discretize it in space in the same form as the HJ operator associated to the FP equation. We use the monotone scheme

$$
\tilde{N}_{n}(u) \equiv \frac{\max \left\{u_{n}-u_{n-1}, 0\right\}^{2}}{h^{2}}+\frac{\max \left\{u_{n}-u_{n+1}, 0\right\}^{2}}{h^{2}}-\frac{1}{\left(1-\rho_{n}\right)^{2}} .
$$

We are then left to solve, at discrete level, the system

$$
\left\{\begin{array}{l}
\rho_{t}+\left(D_{u} N_{n}(u)\right)^{T} \rho=0 \\
\tilde{N}_{n}(u)=0
\end{array}\right.
$$

at each point of the grid. In this way, all the spatial part of the operators was treated. First equation of (5.6) is a time-dependent matrix ODE in $\rho$, whose spatial part has $u_{j}$ 's as parameters (updated by the solution of the second equation). This ODE is supplemented with a discretization of the initial condition for the density, defined in the original continuous Hughes system (5.1). The second equation, the discretized version of the EK equation, is a difference equation in the $u_{j}$ variable, with $\rho_{j}$ as a parameter (updated by the approximation of the solution of the first equation at each time iteration).

Up to now, we have only treated the spatial part of the operators and we have made the choice of discretization with a monotone finite-difference scheme. To solve in time the ODEs in (5.6), we can use standard solvers either explicit or implicit. Since in the practice of the tests presented in this work, the matrix $\left(D_{u} N_{n}(u)\right)^{T}$ is not stiff, we opted for reasons of simplicity and low computing time for an explicit Euler method. In the case of less regular Hamiltonian and consequently less stable systems we recommend the use of Backward Differentiation Formulas (BDF).

For the second equation in (5.6), which contain a high dimensional nonlinear optimization problem, we use a fixed-point iteration as in [32]. More sophisticated techniques can give improved performance for this numerical step, as the policy iteration algorithm [18] or domain decomposition techniques [4, $17]$.

\subsection{Example: 1-D forward-forward mean-field games}

Here, we consider two one-dimensional forward-forward mean-field game problems, see $[1,21,22]$. A special case of such systems is

$$
\left\{\begin{array}{l}
u_{t}+H\left(u_{x}\right)=\varepsilon u_{x x}+g(\rho) \\
\rho_{t}-\left(H^{\prime}\left(u_{x}\right) \rho\right)_{x}=\varepsilon \rho_{x x}
\end{array}\right.
$$

together with the initial-initial conditions

$$
\left\{\begin{array}{l}
u(x, 0)=u_{0}(x) \\
\rho(x, 0)=\rho_{0}(x)
\end{array}\right.
$$


and periodic boundary conditions.

Now, we explain how such systems were treated numerically. MFGs have built-in the adjoint structure we have considered so far, i.e., the FP equation of a MFG system is the adjoint of the linearization of the HJ equation present in the MFG system. Hence, we can use the same type of spatial schemes for the discretization of both the FP and the HJ equation. As described in the previous section, each of the discretizations requires solving an ODE in time. Since we must solve the system of FP coupled to a HJ equation, we treat these ODEs as a single system, and we apply a suitable solver for the time discretization. This manner of solving is possible because we are solving a FFMFG, where both equations evolve forward in time. For backward-forward MFG, either a fixed point iteration between the resolution of the two systems or a simultaneous resolution is required. In the next two problems, we use a monotone finitedifference method for the spatial discretization, as in section 4.1, and an explicit Euler method in time.

For the first problem, we set $H\left(u_{x}\right)=\frac{u_{x}^{2}}{2}, g(\rho)=\ln \rho$, and $\varepsilon=0.01$. We then solve:

$$
\left\{\begin{array}{l}
u_{t}+\frac{u_{x}^{2}}{2}=0.01 u_{x x}+\ln \rho, \\
\rho_{t}-\left(u_{x} \rho\right)_{x}=0.01 \rho_{x x}
\end{array}\right.
$$

with the initial-initial conditions:

$$
\left\{\begin{array}{l}
u_{0}(x)=0.3 \cos (2 \pi x), \\
\rho_{0}(x)=1
\end{array}\right.
$$

We depict the solution of this problem in Figure 1. Here, we see how the oscillations in the evolution of the system reduce illustrating the convergence, for $t \rightarrow+\infty$, to a stationary solution. This convergence for systems as $(5.8)$, for $t \rightarrow+\infty$ was discussed in [22].

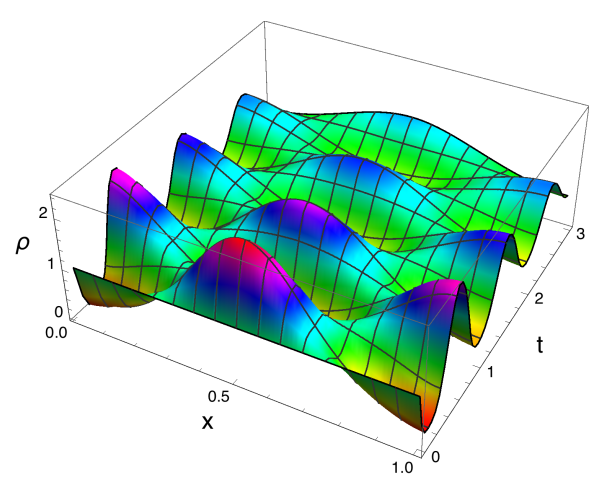

(a) Density

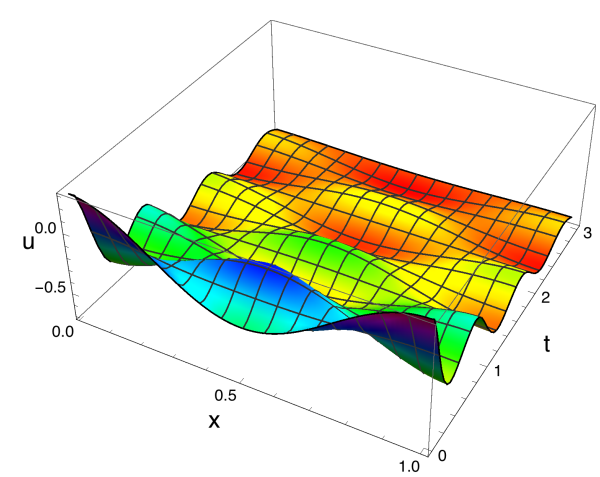

(b) Value function.

Fig. 1: Solutions for $g(\rho)=\ln \rho$.

Now, for the second case, we choose $H\left(u_{x}, \rho\right)=\frac{\left(p+u_{x}\right)^{2}}{2 \rho^{\alpha}}, g(\rho)=\frac{3}{2} \rho^{\alpha}$, and $\varepsilon=0$. This example is a first-order FFMFG with congestion, which is 
equivalent to a system of conservation laws. Setting $v=p+u_{x}$, the equivalent system is

$$
\left\{\begin{array}{l}
v_{t}+\left(\frac{v^{2}}{2 \rho^{\alpha}}-\frac{3}{2} \rho^{\alpha}\right)_{x}=0, \\
\rho_{t}-\left(\rho^{1-\alpha} v\right)_{x}=0 .
\end{array}\right.
$$

For $\alpha=1$, and for the initial-initial conditions

$$
\left\{\begin{array}{l}
u_{0}=-0.5 \frac{\cos (2 \pi x)}{2 \pi}, \\
\rho_{0}=1+0.5 \sin (2 \pi x),
\end{array}\right.
$$

the solution for the density in (5.9) is a traveling wave, depicted in Figure 2. Such failure of convergence is an interesting phenomenon identified in [21] for first-order forward-forward MFGs, and it illustrates an important difference between these two MFG models.

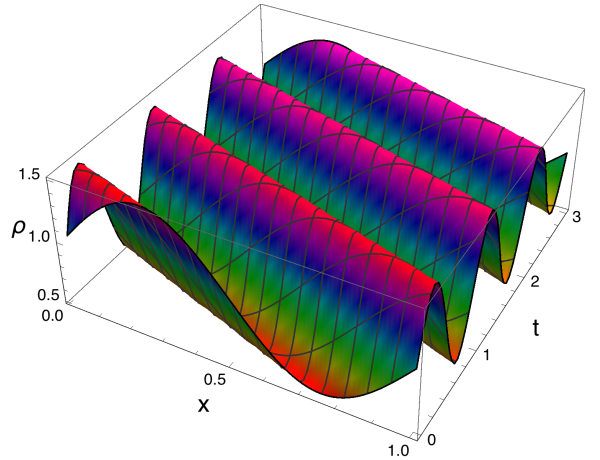

(a) Density.

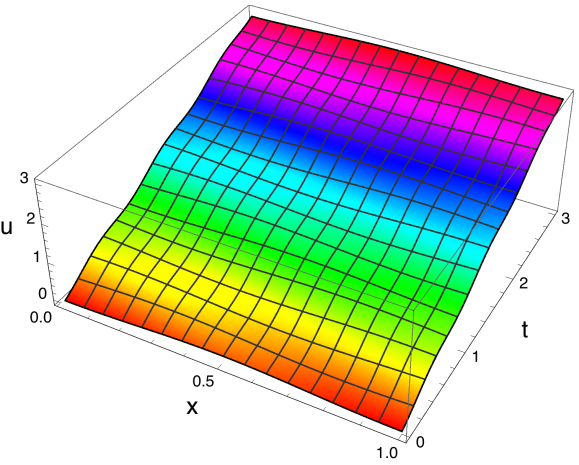

(b) Value function

Fig. 2: Solutions for the FFMFG with congestion.

The simulations corresponding to Figure 1 and Figure 2 were produced with a spatial grid of 80 points, final time $T=3$, and 50 points for the time sample. The simulations run in about one second.

\subsection{Example: Hughes model in 2-D}

In this section, we illustrate our approach for 2-D models. We repeat the steps of section 5.1, with the modification of choosing a monotone finite-difference scheme in 2-D. The domain of our example is a rectangle $[0,3] \times[0,1]$, modeling a room with walls, and an exit on $[2.25,3] \times\{1\}$, corresponding to a typical proportion of the size of a door in a room. We set the value of $u$ to $+\infty$ on all the boundary but on its exit, where we fix it equal to zero. The density is set equal zero on the boundary.

We perform the simulations analogously to the Hughes model in 1-D. We depict the initial condition and its evolution in Figure 3. The spatial grid contains 243 points, and we choose the final time $T=1.0$. The simulation runs in 12.9 seconds. We remark that, at each time iteration for the solution of the Fokker-Planck equation one Eikonal equation in 2-D is being solved. In this example, 83 Eikonal equations were solve and they took $78 \%$ of the total simulation time. 
We end this section by remarking that, in all our simulations both positivity and mass preservation were observed, except when agents are leaving through the boundary; clearly, in the Hughes model, the mass is preserved as long as no agents reach the exit.

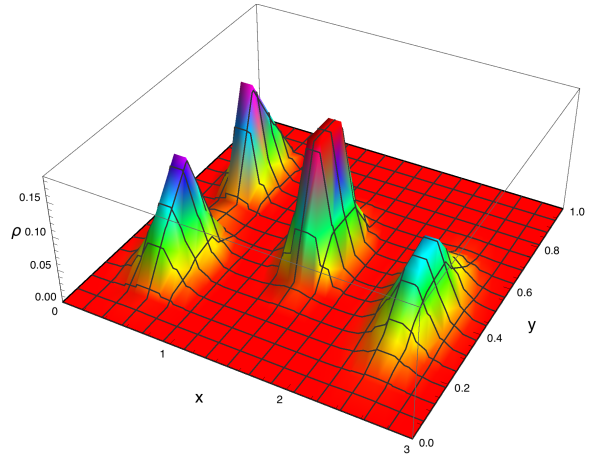

(a) Initial Density.

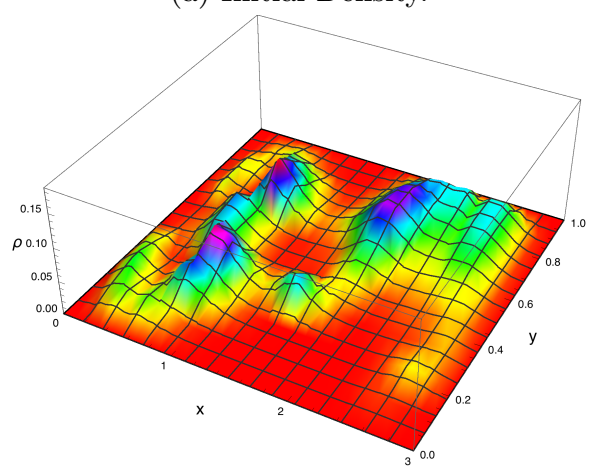

(c) Density at time 0.5 .

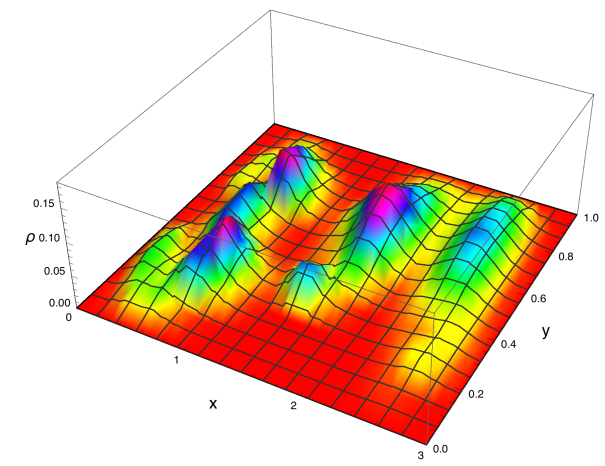

(b) Density at time 0.33 .

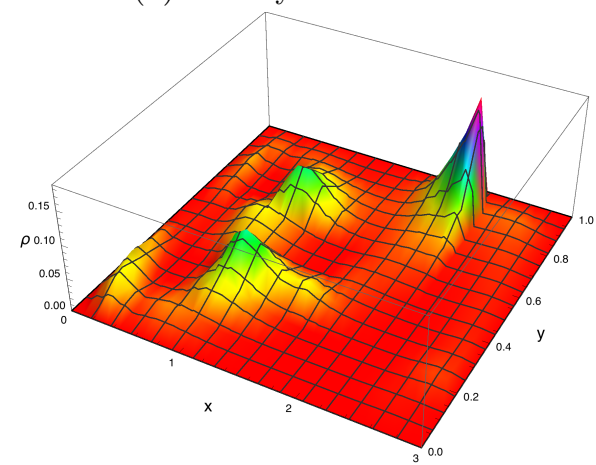

(d) Final density at time 1.0.

Fig. 3: Evolution of the density for the Hughes model.

\section{Conclusions}

In this work, we develop an approach for the approximation of nonlinear FokkerPlanck equations via its adjoint Hamilton-Jacobi operator. Our methodology guarantees that the produced schemes preserve mass and positivity. Consistency is also addressed. We then solve systems of PDEs with a Fokker-Planck equation coupled to a Hamilton-Jacobi equation. Our methods apply to a broad range of problems where a measure-potential structure appears, including mean-field games, crowd and traffic models, and chemotaxis.

In future work, we plan to address different schemes developed for HJ equations to study FP equations. Originally, schemes such as the Discontinuous Galerkin or ENO schemes were developed for conservation laws and later gave rise to effective numerical schemes for HJ equations. With our methods we can reverse this process by starting with schemes for Hamilton-Jacobi equations and then deriving schemes for FP equations. Nevertheless, it is clear that, without monotonicity and stability properties, results for the convergence of such 
schemes are difficult to achieve.

\section{References}

[1] Y. Achdou and I. Capuzzo Dolcetta. Mean field games: Numerical methods. SIAM Journal of Numerical Analysis, 48-3:1136-1162, 2010.

[2] Y. Achdou, F. Camilli, and I. Capuzzo Dolcetta. Mean field games: convergence of a finite difference method. SIAM J. Numer. Anal., 51(5):2585$2612,2013$.

[3] M. Bossy, E. Gobet, and D. Talay. A symmetrized Euler scheme for an efficient approximation of reflected diffusions. J. Appl. Probab., 41(3):877889, 2004.

[4] S. Cacace, E. Cristiani, M. Falcone, and A. Picarelli. A patchy dynamic programming scheme for a class of hamilton-jacobi-bellman equations. SIAM Journal on Scientific Computing, 34(5):A2625-A2649, 2012.

[5] F. Cagnetti, D. Gomes, and H. V. Tran. Aubry-Mather measures in the nonconvex setting. SIAM J. Math. Anal., 43(6):2601-2629, 2011. ISSN 0036-1410. doi: 10.1137/100817656. URL http://dx.doi.org/10.1137/ 100817656.

[6] F. Cagnetti, D. Gomes, and H. V. Tran. Adjoint methods for obstacle problems and weakly coupled systems of PDE. ESAIM Control Optim. Calc. Var., 19(3):754-779, 2013. ISSN 1292-8119. doi: 10.1051/cocv/ 2012032. URL http://dx.doi.org/10.1051/cocv/2012032.

[7] F. Cagnetti, D. Gomes, and H. V. Tran. Convergence of a semidiscretization scheme for the Hamilton-Jacobi equation: a new approach with the adjoint method. Appl. Numer. Math., 73:2-15, 2013. ISSN 01689274. doi: 10.1016/j.apnum.2013.05.004. URL http://dx.doi.org/10 . 1016/j . apnum. 2013.05.004.

[8] F. Cagnetti, D. Gomes, H. Mitake, and H. V. Tran. A new method for large time behavior of degenerate viscous Hamilton-Jacobi equations with convex Hamiltonians. Ann. Inst. H. Poincaré Anal. Non Linéaire, 32(1): 183-200, 2015. ISSN 0294-1449. doi: 10.1016/j.anihpc.2013.10.005. URL http://dx.doi.org/10.1016/j.anihpc.2013.10.005.

[9] E. Carlini and F. J. Silva. Semi-lagrangian schemes for mean field game models. In Decision and Control (CDC), 2013 IEEE 52nd Annual Conference on, pages 3115-3120. IEEE, 2013.

[10] E. Carlini and F. J. Silva. A fully discrete semi-Lagrangian scheme for a first order mean field game problem. SIAM J. Numer. Anal., 52(1):45-67, 2014. ISSN 0036-1429. doi: 10.1137/120902987. URL http://dx.doi . org/10.1137/120902987.

[11] E. Carlini and F. J. Silva. A semi-Lagrangian scheme for a degenerate second order mean field game system. Discrete Contin. Dyn. Syst., 35(9): 4269-4292, 2015. ISSN 1078-0947. doi: 10.3934/dcds.2015.35.4269. URL http://dx.doi.org/10.3934/dcds.2015.35.4269. 
[12] E. Carlini and F. J. Silva. A semi-lagrangian scheme for the fokkerplanck equation. IFAC-PapersOnLine, 49(8):272 - 277, 2016. ISSN 24058963. doi: http://dx.doi.org/10.1016/j.ifacol.2016.07.453. URL http: //www.sciencedirect.com/science/article/pii/S2405896316306619.

[13] E. Carlini, A. Festa, F. J. Silva, and M.-T. Wolfram. A Semi-Lagrangian scheme for a modified version of the Hughes' model for pedestrian flow. Dyn. Games Appl., 2016. doi:10.1007/s13235-016-0202-6.

[14] P.H. Chavanis. Nonlinear mean field fokker-planck equations. application to the chemotaxis of biological populations. The European Physical Journal B-Condensed Matter and Complex Systems, 62(2):179-208, 2008.

[15] L. C. Evans. Adjoint and compensated compactness methods for hamiltonjacobi pde. Archive for rational mechanics and analysis, 197(3):1053-1088, 2010.

[16] M. Falcone and R. Ferretti. Semi-Lagrangian approximation schemes for linear and Hamilton-Jacobi equations. Society for Industrial and Applied Mathematics (SIAM), Philadelphia, PA, 2014. ISBN 978-1-611973-04-4.

[17] A. Festa. Reconstruction of independent sub-domains for a class of hamilton-jacobi equations and application to parallel computing. ESAIM: Mathematical Modelling and Numerical Analysis, 50(4):1223-1240, 2016.

[18] A. Festa. Domain decomposition based parallel howard's algorithm. Mathematics and Computers in Simulation, 2017. ISSN 0378-4754. doi: https://doi.org/10.1016/j.matcom.2017.04.008. URL http://www . sciencedirect.com/science/article/pii/S0378475417301684.

[19] E. Gobet. Weak approximation of killed diffusion using Euler schemes. Stochastic Process. Appl., 87(2):167-197, 2000.

[20] D. Gomes, E. Pimentel, and V. Voskanyan. Regularity theory for mean-field game systems. SpringerBriefs in mathematics, 2016.

[21] D. A. Gomes and M. Sedjro. One-dimensional forward-forward mean-field games with congestion. Preprint - Submitted to Discrete and Continuous Dynamical Systems-Series S., 2017.

[22] D. A. Gomes, L. Nurbekyan, and M. Sedjro. One-dimensional forwardforward mean-field games. Appl. Math. Optim., 74(3):619-642, 2016. ISSN 0095-4616. doi: 10.1007/s00245-016-9384-y. URL http://dx.doi.org/ 10.1007/s00245-016-9384-y.

[23] T. Goudon and M. Saad. On a fokker-planck equation arising in population dynamics. Rev. Mat. Complut, 11(2):353-372, 1998.

[24] O. Guéant. Mean field games and applications to economics. PhD thesis, Université Paris-Dauphine, 2009.

[25] R. L. Hughes. A continuum theory for the flow of pedestrians. Transportation Research Part B: Methodological, 36(6):507-535, 2002. 
[26] B. Jourdain and S. Méléard. Propagation of chaos and fluctuations for a moderate model with smooth initial data. Ann. Inst. H. Poincaré Probab. Statist., 34(6):727-766, 1998. ISSN 0246-0203. doi: 10.1016/S0246-0203(99)80002-8. URL http://dx.doi.org/10.1016/ S0246-0203(99)80002-8.

[27] A. Lachapelle and M.-T. Wolfram. On a mean field game approach modeling congestion and aversion in pedestrian crowds. Transportation Research Part B: Methodological, 45:1572-1589, 2011.

[28] A. Lachapelle, J. Salomon, and G. Turinici. Computation of mean field equilibria in economics. Mathematical Models and Methods in Applied Sciences, 20-4:567-588, 2010.

[29] H. P. McKean, Jr. A class of Markov processes associated with nonlinear parabolic equations. Proc. Nat. Acad. Sci. U.S.A., 56:1907-1911, 1966. ISSN 0027-8424.

[30] H. P. McKean, Jr. Propagation of chaos for a class of non-linear parabolic equations. In Stochastic Differential Equations (Lecture Series in Differential Equations, Session 7, Catholic Univ., 1967), pages 41-57. Air Force Office Sci. Res., Arlington, Va., 1967.

[31] S. Méléard. Asymptotic behaviour of some interacting particle systems; McKean-Vlasov and Boltzmann models. In Probabilistic models for nonlinear partial differential equations (Montecatini Terme, 1995), volume 1627 of Lecture Notes in Math., pages 42-95. Springer, Berlin, 1996. doi: 10.1007/BFb0093177. URL http://dx.doi.org/10.1007/BFb0093177.

[32] A. M. Oberman. Convergent difference schemes for degenerate elliptic and parabolic equations: Hamilton-Jacobi equations and free boundary problems. SIAM J. Numer. Anal., 44(2):879-895, 2006. ISSN 0036-1429. doi: 10.1137/S0036142903435235. URL http://dx.doi.org/10.1137/ S0036142903435235.

[33] A.-S. Sznitman. Topics in propagation of chaos. In École d'Été de Probabilités de Saint-Flour XIX-1989, volume 1464 of Lecture Notes in Math., pages 165-251. Springer, Berlin, 1991. doi: 10.1007/BFb0085169. URL http://dx.doi.org/10.1007/BFb0085169.

[34] A. Tosin, A. Festa, and M-T. Wolfram. Kinetic description of collision avoidance in pedestrian crowds by sidestepping. arXiv preprint arXiv:1610.05056, 2016.

[35] H. V. Tran. Adjoint methods for static Hamilton-Jacobi equations. Calc. Var. Partial Differential Equations, 41(3-4):301-319, 2011. ISSN 09442669. doi: 10.1007/s00526-010-0363-x. URL http://dx.doi.org/10. $1007 / \mathrm{s} 00526-010-0363-\mathrm{x}$. 